Research Article

\title{
Ectopic Pregnancy in Tigray, Ethiopia: A Cross-Sectional Survey of Prevalence, Management Outcomes, and Associated Factors
}

\author{
Elsa Tesfa Berhe $\mathbb{D},{ }^{1}$ Kalayu Kiros $\mathbb{D},{ }^{2}$ Merhawit Gebremeskel Hagos $\mathbb{D},{ }^{3}$

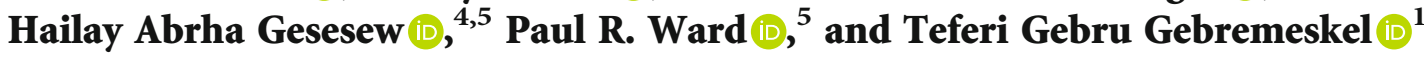 \\ ${ }^{1}$ Department of Reproductive Health, College of Health Sciences, Aksum University, Aksum, Ethiopia \\ ${ }^{2}$ School of Medicine, College of Medicine and Health Science, Aksum University, Aksum, Ethiopia \\ ${ }^{3}$ Department of Midwifery, College of Health Sciences, Aksum University, Aksum, Ethiopia \\ ${ }^{4}$ Discipline of Public Health, Flinders University, Adelaide, Australia \\ ${ }^{5}$ Department of Epidemiology, College of Health Sciences, Mekele University, Mekele, Ethiopia
}

Correspondence should be addressed to Teferi Gebru Gebremeskel; teferigebru12@gmail.com

Received 5 July 2021; Accepted 15 November 2021; Published 30 November 2021

Academic Editor: I. Bellos

Copyright (c) 2021 Elsa Tesfa Berhe et al. This is an open access article distributed under the Creative Commons Attribution License, which permits unrestricted use, distribution, and reproduction in any medium, provided the original work is properly cited.

Background. Ectopic pregnancy is a neglected and challenging gynecologic problem in developing countries including Ethiopia. Objective. The present study is aimed at assessing the prevalence of ectopic pregnancy, its management outcomes, and factors associated with management outcomes in Tigray, North Ethiopia. Methods. We employed a four-year retrospective crosssectional study from September 2015 to August 2019. We extracted data about all pregnant mothers who were admitted and managed for EPs in Axum, Tigray. Ectopic pregnancy and its outcomes (favorable and unfavorable) were the dependent variables, and age, residence, ethnicity, religion, parity, history of abortion, history of EP, pelvic infections, history of surgical procedures, and use contraceptives were the independent variables. We employed descriptive statistics and bivariate and multivariate logistic regression analyses using SPSS. Ethical clearance was obtained from Axum University, Tigray, Ethiopia. Results. The overall prevalence of ectopic pregnancy was $0.52 \%$ of total deliveries, which equates to $1: 193$ deliveries. Surgery for ectopic pregnancy accounts for $7.6 \%$ of all gynecological surgeries. Most participants were in the age group 26-30 years and lived in rural areas. Among the different EP implantation sites, most cases (92.4\%) occurred in the fallopian tube, followed by $5.1 \%$ in the ovary and $2.5 \%$ in abdominal EPs. Surgical management (laparotomy) was undertaken for all the 79 women diagnosed with EPs, including laparotomy (100\%), salpingo-oophorectomy (17.7\%), salpingectomy (73.9\%), oophorectomy (3.4\%), cornual resection $(2.5 \%)$, and removal of concepts tissue 2.5 . The record reports that intraoperative procedure was correctly managed for 47 (59.5\%) women but the condition of EP procedure was ruptured for about two-thirds (63.3\%) of the women. Thirty (38\%) patients had developed some complications after surgery including anemia (hemoglobin $<10.5)(n=12)$, fever $(n=10)$, wound infection $(n=2)$, and pneumonia $(n=2)$. Women who were from urban (AOR $=11.2,95 \%$ CI: $2.65-$ 47.2) and who had normal hemoglobin at presentation $(\mathrm{AOR}=9.94,95 \%$ CI: 2.03-48.7) were associated with favorable maternal outcomes. Conclusions. More than one-third of women with ectopic pregnancies had an unfavorable maternal outcome, which was higher among rural residents and anemic mothers. Women living in rural areas and anemia during pregnancy should seek special attention in the management of EPs. We also recommend improving the data management of hospitals in Ethiopia. 


\section{Introduction}

Ectopic pregnancy (EP), an extrauterine pregnancy, is one in which the blastocyst implants anywhere other than the endometrial lining of the uterine cavity [1]. EP is a global problem, and the most common life-threatening emergency in gynecological practice and is a medical emergency that requires immediate treatment [2]. This complication is prevalent in low- and middle-income countries, where the majority of mothers present late after their onset of illness with hemodynamic instability and tubal rupture [3]. A five-year retrospective study (2009-2013) in the University of Benin, Benin, found that $89 \%$ of all pregnant patients with hemodynamic instability and $88 \%$ of cases of tubal rupture have ruptured EP [4].

The incidence of EP has tripled in most industrialized countries over the past 3 decades, and the annual incidence rates vary between 100 and 175 per 100,000 women age between 15 and 44 [5]. The incidence rates of EP in African countries range between 0.5 and $2.3 \%$ of live births [6]. In Ethiopia, the magnitude of EP among total deliveries was $0.82 \%$ [7]. Globally, EP accounts for almost $2 \%$ of all pregnancies, $0.25-2 \%$ of all pregnancy-related complications, and $9 \%$ of all pregnancy-related deaths $[8,9]$.

EP is diagnosed with a "classic" triad of delayed menstruation, pain, vaginal bleeding or spotting, and a positive pregnancy test. However, only $50 \%$ of patients present with typical symptomatology [10]. The exact etiology of EP is elusive but the factors which predispose women to EP include age, abortion status, ovulation stimulation, pelvic infection, tubal surgeries, and use of modern contraceptives [8, 11]. For example, a ten-year cross-sectional study in Nigeria (2002-2011) showed that the majority of cases were between 26 and 30 years, were married, multiparous, and had a previous history of induced abortions [11].

The clinical presentation of EP varies from asymptomatic to acute abdominal pain and may present with hypovolemia and shock $[8,11]$. A four-year cross-sectional study conducted in South Africa showed abdominal pain was the main complaint of patients, accounting for $81.1 \%$ of the recorded symptoms, and more than one-third of patients had signs of shock when they presented to the hospital [12]. Most morbidity outcomes of EP included anemia, blood transfusion (78\%), and wound infection (2.1\%) [13]. In Ethiopia, EP accounts for $3.74 \%$ of gynecological surgeries, and the majority (51.9\%) of mothers were in the age group of 25-29 years [7]. Abdominal pain, vaginal bleeding, and amenorrhea were the most common symptoms at presentation, and most mothers (61\%) presented with ruptured EP [7].

EP cause has a direct impact on women's reproductive health and is a significant issue for the community [10]. EP remains a significant cause of maternal morbidity and mortality in developing countries [11]. However, the importance of EP in developing countries has received reduced attention, partly because of its late presentations of patients with ruptured EP, poor diagnostic tools, limited capacity to handle emergencies, and consequent burden of increased maternal morbidity and mortality [7]. Importantly, there is little evidence on EP in Africa in general and north Ethiopia in particular. The present study was conducted to perform a comprehensive assessment including determining the prevalence of EP, exploring management outcomes, and identifying underlying risk factors using a case of Aksum University Comprehensive and Specialized Hospital in Tigray, Ethiopia.

\section{Materials and Methods}

2.1. Study Design, Population, and Setting. We have performed a health facility-based retrospective cross-sectional study dated from September 2015 to August 2019. Records of total deliveries and gynecological surgeries of all pregnant mothers who were diagnosed after a clinical examination, investigations, and treatment were included in the study.

The study was conducted in Aksum University Comprehensive and Specialized Hospital (AKUCSH) and Saint Marries General Hospital in Tigray regional state, northern Ethiopia. Aksum is the capital of the central zone of Tigray which is located $1024 \mathrm{~km}$ north of Addis Ababa and $247 \mathrm{~km}$ from Mekele, the capital city of the Tigray. According to the plan and finance office of Aksum town (personal communication, 2020), the total population size is 77,723 of which 38,402 are males and 39,321 are females. Axum city has five kebeles, one referral and teaching hospital, one general hospital, two health centers, four health posts, and 10 different level private clinics.

AKUCSH started as a referral hospital in 2014 for serving an estimated 3.6 million population in its catchment areas. Currently, the hospital has around 760 health workers including 19 specialists. The hospital provides emergency, inpatient, and outpatient services at different departments. Currently, the Gynecology \& Obstetrics (GYN/OBS) department has two gynecologists/obstetricians, 12 GPs, and 49 midwives. The hospital has 177 beds in four wards: medical, pediatrics, surgical, gynecology, and obstetrics wards. Of these, the maternity ward has 42 beds, and delivery and labor wards have 13 beds. Saint Marries General Hospital, the other study setting, was established in 1961 and has 368 health care workers including 17 general practitioners (GPs). In the GYN/OBS department, there are one gynecologist/obstetrician, one Integrated Emergency Surgeon and Obstetrician (IESO), and 18 midwives. The GYN/OBS department has 28 beds and delivers similar services to AUCSH.

All total deliveries and gynecological surgeries to Aksum University Comprehensive Referral Hospital and Saint Marries General Hospital from September 1, 2015 to August 6, 2019 were reviewed.

2.2. Variables and Data Collection Process. In the dependent variable of the study presence of favorable/unfavorable outcomes of EP, Elements of management outcomes included recorded outcomes including side effects of EP, hemoperitoneum collection, estimation of blood loss, blood transfusion, postoperative complications, duration of hospital stay, and maternal outcomes. An unfavorable outcome was assumed if one of the following identified: unstable vital sign, blood transfusion, prolonged hospital stay, postoperative 
TABLE 1: Sociodemographic and gynecologic characteristic distribution of women admitted with EP in Aksum (AKU CHS CSH and Saint Marries General Hospital) in Tigray, North Ethiopia.

\begin{tabular}{|c|c|c|c|}
\hline Variables & & Frequency, $n=79$ & Percent $(\%)$ \\
\hline \multirow{5}{*}{ Age } & $\leq 20$ years & 9 & $11.4 \%$ \\
\hline & $21-25$ years & 11 & $13.9 \%$ \\
\hline & $26-30$ years & 33 & $41.8 \%$ \\
\hline & $31-35$ years & 14 & $17.7 \%$ \\
\hline & $\geq 36$ years & 12 & $15.2 \%$ \\
\hline \multirow{2}{*}{ Residence } & Urban & 39 & $49.4 \%$ \\
\hline & Rural & 40 & $50.6 \%$ \\
\hline \multirow{2}{*}{ Ethnicity } & Tigrai & 77 & $97.5 \%$ \\
\hline & Amhara & 2 & $2.5 \%$ \\
\hline \multirow{2}{*}{ Religion } & Orthodox & 77 & $97.5 \%$ \\
\hline & Muslim & 2 & $2.5 \%$ \\
\hline \multirow{3}{*}{ Parity } & Nulliparous & 22 & $27.8 \%$ \\
\hline & Primipara & 26 & $32.9 \%$ \\
\hline & Multipara & 31 & $39.2 \%$ \\
\hline \multirow{3}{*}{ Previous history of abortion } & No abortion & 47 & $59.5 \%$ \\
\hline & History of one or two abortions & 28 & $35.4 \%$ \\
\hline & Three and above abortion & 4 & $5.1 \%$ \\
\hline \multirow{2}{*}{ Previous history of EP } & No history of EP & 73 & $92.4 \%$ \\
\hline & One previous EP & 6 & $7.6 \%$ \\
\hline \multirow{2}{*}{ Previous history of pelvic infections } & No history of pelvic infection & 55 & $69.6 \%$ \\
\hline & Had a history of pelvic infection at least once & 24 & $30.4 \%$ \\
\hline \multirow{2}{*}{ Previous history of surgery } & No history of surgery & 67 & $84.8 \% \mathrm{~s}$ \\
\hline & Had a history of surgery at least once & 12 & $15.2 \%$ \\
\hline \multirow{2}{*}{ History of contraceptive use } & No history of contraceptive use & 38 & $48.1 \%$ \\
\hline & History of contraceptive use at least once & 41 & $51.9 \%$ \\
\hline
\end{tabular}

complications, and death. Anemia was assumed hemoglobin value of $<10.5 \mathrm{mg} / \mathrm{dl}$. Unstable vital sign was assumed if one of the following occurred: $\mathrm{SBP}<90 \mathrm{~mm} \mathrm{Hg}$ and/or DBP $<$ $60 \mathrm{~mm} \mathrm{Hg}$, pulse rate $>100$ beats per minute, respiratory rate $>30$ breaths per minute, and/or temperature $>37.5^{\circ} \mathrm{C}$. A kebele is the smallest administrative unit of Ethiopia, similar to a ward, a neighborhood or a localized, and delimited group of people. It is part of districts, itself usually part of a zone, which in turn are grouped into one of the ten regions or two chartered cities that comprise the Federal Democratic Republic of Ethiopia [14]. Each kebele consists of at least 500 families or the equivalent of 3,500 to 4,000 persons. There is at least one in every town with more than 2,000 populations. A district representative had jurisdiction over to ward [15].

EPs were managed in the hospitals by laparotomy (most cases with pfannenstiel incision, minilaparotomy never tried, and medical management not available) and if patient had pneumonia complications, spinal anesthesia (SA), and prophylactic antibiotics that are routinely given. Data were retrieved on EP status, parity, clinical presentation, risk factors, findings at laparotomy, and the outcome of the treatment from the maternity ward, labor, and delivery ward and operation room registration books and medical records. Records that did not include the EP status and one of the aforementioned management outcomes were excluded from data analysis. In addition, we extracted data on relevant sociodemographic characteristics, parity, abortion, sexually transmitted infection, contraceptive use, obstetric and gynecologic surgery, and previous EPs.

The data extraction sheet was prepared in English and then translated into the local language (Tigrigna) and back to English by a professional translator. To establish face validity and translation quality, the data extraction sheet was tested on $5 \%$ of the total sample size determined for this study (participants were outside of study site) by data collectors and supervisors during training. A few questions, language clarity, and information were revised, and the data extraction sheet was finalized for the study.

Data were extracted by four BSc nurses and supervised by one GP. We delivered training for data collectors and supervisors on the study's objective, ethical procedures, and data collection process.

2.3. Data Analysis Process. Data were initially checked manually for completeness and consistency by the supervisor and principal investigator during the fieldwork and rechecked before data entry. Then, data were coded, entered, and cleaned using Epi Info version 7 to export to SPSS version 20.0 software for analysis and interpretation. 
TABLE 2: Clinical presentation of patients of women admitted with ectopic pregnancy in Aksum (AKU CHS CSH and Saint Marries General Hospital) in Tigray, North Ethiopia, $(n=79)$.

\begin{tabular}{|c|c|c|c|}
\hline & Characteristics & Frequency & Percent (\%) \\
\hline \multirow{2}{*}{ Time of presentation } & Within 24 hours of the onset of illness & 19 & $24.1 \%$ \\
\hline & More than 24 hours of the onset of illness & 60 & $75.9 \%$ \\
\hline \multirow{3}{*}{ Vital sign at presentation } & Stable & 51 & $64.6 \%$ \\
\hline & Unstable & 28 & $35.4 \%$ \\
\hline & Abdominal pain & 77 & $97.5 \%$ \\
\hline \multirow{3}{*}{ Presenting symptoms* } & Vaginal bleeding & 64 & $81 \%$ \\
\hline & Amenorrhea & 45 & $57 \%$ \\
\hline & Vomiting & 10 & $12.7 \%$ \\
\hline \multirow{5}{*}{ Physical examination finding* } & Pale conjunctiva and/or palm & 34 & $43 \%$ \\
\hline & Abdominal tenderness & 75 & $94.9 \%$ \\
\hline & Cervical motion tenderness & 24 & $30.4 \%$ \\
\hline & Adnexal mass & 2 & $2.5 \%$ \\
\hline & Adnexal tenderness & 12 & $15.2 \%$ \\
\hline \multirow{2}{*}{ Urine HCG result } & Positive & 77 & $97 . \%$ \\
\hline & Negative & 2 & $2.5 \%$ \\
\hline \multirow{2}{*}{ Level of hemoglobin at presentation } & Hemoglobin $>10.5$ at presentation & 33 & $41.8 \%$ \\
\hline & Hemoglobin $<10.5$ at presentation & 46 & $58.2 \%$ \\
\hline \multirow{4}{*}{ Diagnosis } & Clinical only & 4 & $5.1 \%$ \\
\hline & Ultrasound only & 2 & $2.5 \%$ \\
\hline & Intraoperative only & 2 & $2.5 \%$ \\
\hline & Clinical and ultrasound & 71 & $89.9 \%$ \\
\hline \multirow{3}{*}{ Gestational age } & Less than 7 weeks & 45 & $57 \%$ \\
\hline & 7-9 weeks & 18 & $22.8 \%$ \\
\hline & More than 9 weeks & 16 & $20.2 \%$ \\
\hline
\end{tabular}

${ }^{*}$ Participants reported more than one symptom or sign.

TABLE 3: Yearly distribution of ectopic pregnancy per number of registered deliveries in Aksum (AKUCSH and Saint Marries General Hospital), Tigray, Ethiopia, 2015-19.

\begin{tabular}{lccc}
\hline Period & Total registered deliveries & Total EP & Percent (\%) \\
\hline 2015 & 2779 & 20 & 0.72 \\
2016 & 3169 & 17 & 0.54 \\
2017 & 3761 & 16 & 0.42 \\
2018 & 5495 & 26 & 0.47 \\
Total & 15204 & 79 & 0.52 \\
\hline
\end{tabular}

EP: ectopic pregnancy.

Descriptive statistics were performed, and data were described using tables. We applied bivariate and multivariate logistic regression analysis to identify variables associated with the management outcomes of EP. Variables with a $p$ $<0.25$ in the bivariate logistic regression analyses were entered into the multivariate logistic regression analyses. Chi-square tests were applied before bivariate logistic regression to check variables with small cell sizes to merge into related categories. Goodness of fit tests was conducted using Hosmer and Lemeshow Chi-square tests. Multicollinearity was assessed using collinearity diagnosis to test correlation among predictor variables. Finally, independently associated variables were considered if $p<0.05$ in multiple logistic regression models. The strength of association was described using the adjusted odds ratio (AOR) and 95\% confidence interval $(\mathrm{CI})$.

\section{Results}

3.1. Sociodemographic and Gynecologic Characteristics of EP Women. Table 1 describes the sociodemographic and gynecologic characteristics of the study participants. The mean age of the women diagnosed with EP was 29 years $(\mathrm{SD} \pm 5.96)$ years ranging between 19 and 45 years. A significant proportion of women (42\%) with EP was in the age group of 26 to 30 years, and half $(50.6 \%)$ of the study participants came from the rural area. Of the total 79 women admitted with EP, about $39 \%$ of them were multipara, $8 \%$ had a previous history of EP, and $4 \%$ had previous Caesarean section history.

\subsection{Clinical Presentation of Women Diagnosed with Ectopic} Pregnancy. Table 2 presents the clinical presentation of women diagnosed with EP. Three quarters (76\%) of the women diagnosed with EP presented after 24 hours of the onset of their complications. Two thirds (65\%) of them presented with stable vital signs at presentation, almost all with 
TABle 4: Management outcome patients admitted with ectopic pregnancy in Aksum, Tigray, North Ethiopia $(n=79)$.

\begin{tabular}{|c|c|c|c|}
\hline & Characteristics & Frequency & Percent (\%) \\
\hline \multirow{2}{*}{ Side of EP } & Right side & 47 & $59.5 \%$ \\
\hline & Left side & 32 & $40.5 \%$ \\
\hline \multirow{2}{*}{ Condition of EP } & Ruptured & 50 & $63.3 \%$ \\
\hline & Unruptured & 29 & $36.7 \%$ \\
\hline \multirow{4}{*}{ Hem peritoneum collection } & Less than $500 \mathrm{ml}$ & 34 & $43 \%$ \\
\hline & $\geq 500 \mathrm{ml}$ & 18 & $22.8 \%$ \\
\hline & No hem peritoneum & 16 & $20.3 \%$ \\
\hline & Not documented & 11 & $13.9 \%$ \\
\hline \multirow{4}{*}{ Estimated blood loss } & Less than $500 \mathrm{ml}$ & 28 & $35.4 \%$ \\
\hline & $500 \mathrm{ml}-1000 \mathrm{ml}$ & 10 & $12.7 \%$ \\
\hline & More than $1000 \mathrm{ml}$ & 6 & $7.6 \%$ \\
\hline & Not documented & 35 & $44.3 \%$ \\
\hline \multirow{4}{*}{ Transfuse for blood } & Not transfused & 52 & $65.9 \%$ \\
\hline & 2 units & 20 & $25.3 \%$ \\
\hline & 3 units & 5 & $6.3 \%$ \\
\hline & 4 units and above & 2 & $2.5 \%$ \\
\hline \multirow{5}{*}{ Postoperative complications } & No complication & 49 & $62 \%$ \\
\hline & Anemia & 12 & $15.2 \%$ \\
\hline & Fever & 10 & $12.7 \%$ \\
\hline & Wound infection & 6 & $7.6 \%$ \\
\hline & Pneumonia & 2 & $2.5 \%$ \\
\hline \multirow{3}{*}{ Duration of hospital stay } & Less than 7 days & 53 & $67.1 \%$ \\
\hline & 7-14 days & 24 & $30.4 \%$ \\
\hline & 14 days -1 month & 2 & $2.5 \%$ \\
\hline \multirow{2}{*}{ Maternal outcome } & Favorable & 49 & $62 \%$ \\
\hline & Unfavorable & 30 & $38 \%$ \\
\hline
\end{tabular}

abdominal pain (98\%) and approximately 1 in 10 with vomiting (13\%). Anemia was also diagnosed in more than half $(58.2 \%)$ of the women with EP.

\subsection{Prevalence of Ectopic Pregnancy Out of Total Deliveries.} The trends of EP over time are presented in Table 3. A total of 15,204 deliveries and 1043 gynecological surgeries were recorded in both hospitals from 2015 to 19. Eighty-three patients were admitted with the diagnosis of EPs but records of 79 of the patients were eligible for data analysis. The overall prevalence of EP was found to be $0.52 \%(79 / 15,204)$ deliveries, 1:193 deliveries. The surgery for EP accounts for $7.6 \%$ (79 of 1,043) of all gynecological surgeries. The highest prevalence of $0.72 \%$ (20 out of 2779 ) was documented in 2015, and the lowest was 0.42 (16 out of 2450) recorded in 2017.

3.4. Management Outcomes of Ectopic Pregnancy. Table 4 presents details of the management outcomes of EP. Among the different EP implantation sites, most cases (92.4\%) occurred in the fallopian tube, followed by $5.1 \%$ in the ovary, and $2.5 \%$ in abdominal EPs. Surgical management was undertaken for all the 79 women diagnosed with EPs, including laparotomy (68.4\%), salpingo-oophorectomy (17.7\%), salpingectomy $(8.9 \%)$, oophorectomy $(2.5 \%)$, and cornual resection $(2.5 \%)$. The intraoperative finding was intact tube in 47 (59.5\%) cases and ruptured in the rest but the condition of EP procedure was ruptured for about twothirds $(63.3 \%)$ of the women.

Most patients (67.1\%) were admitted for less than a week. Thirty (38\%) patients had developed some complications after surgery including anemia $(n=12)$, fever $(n=10$ ) , wound infection $(n=2)$, and pneumonia $(n=2)$.

3.5. Factors Associated with Management Outcome of Ectopic Pregnancy. Residence (urban or rural), previous history of abortions, previous history of surgery, presentation of symptoms from the onset of illness, vital signs at presentation, gestational age, and level of hemoglobin at presentation were the candidate variables for multiple logistic regressions. Only residence and level of hemoglobin at presentation were found to be statistically significant EP management outcomes. Mothers who came from urban areas were 11 times $(\mathrm{AOR}=11.2,95 \% \mathrm{CI}: 2.7-47.2)$ more likely to have favorable outcomes than those coming from rural areas. Mothers who presented with normal hemoglobin were about 10 times (AOR $=9.9,95 \%$ CI: 2.03-48.7) more likely to have a favorable outcome than mothers presented with anemia (see Table 5). 
TABLE 5: Factors associated with maternal outcome among women admitted with ectopic pregnancy in Aksum, Tigray, North Ethiopia $(n=79)$.

\begin{tabular}{|c|c|c|c|c|}
\hline \multirow{2}{*}{ Variable } & \multicolumn{2}{|c|}{ EP management outcome } & \multirow{2}{*}{ COR 95\% CI } & \multirow{2}{*}{ AOR 95\% CI } \\
\hline & Favorable & Unfavorable & & \\
\hline \multicolumn{5}{|l|}{ Residence } \\
\hline Rural & 17 & 23 & 1 & 1 \\
\hline Urban & 32 & 7 & $6.19(2.21-17.3)$ & $11.2(2.7-47.2)^{* *}$ \\
\hline \multicolumn{5}{|l|}{ History of abortion } \\
\hline No & 26 & 21 & 1 & 1 \\
\hline Yes & 23 & 9 & $2.06(1.07-5.4)$ & $4.25(1.03-19.5)$ \\
\hline \multicolumn{5}{|l|}{ Vital sign } \\
\hline Unstable & 12 & 16 & 1 & 1 \\
\hline Stable & 37 & 14 & $3.52(1.34-9.3)$ & $1.56(0.4-5.5)$ \\
\hline \multicolumn{5}{|l|}{ History of surgery } \\
\hline Yes & 6 & 6 & $0.56(0.2-0.9)$ & $2.03(0.4-10.9)$ \\
\hline No & 43 & 24 & 1 & 1 \\
\hline \multicolumn{5}{|l|}{ Hemoglobin } \\
\hline Anemic & 22 & 24 & 1 & 1 \\
\hline Nonanemic & 27 & 6 & $4.9(1.7-4.1)$ & $9.9(2.03-48.7)^{* *}$ \\
\hline \multicolumn{5}{|c|}{ Presentation of illness } \\
\hline Within $24 \mathrm{hr}$ & 10 & 9 & $0.6(0.2-0.9)$ & $0.3(0.06-1.3)$ \\
\hline More than $24 \mathrm{hr}$ & 39 & 21 & 1 & 1 \\
\hline \multicolumn{5}{|l|}{ Gestational age } \\
\hline$<7$ weeks & 31 & 14 & $2.2(1.6-7.1)$ & $1.2(0.2-6.7)$ \\
\hline 7-9 weeks & 10 & 8 & $1.3(1.3-4.8)$ & $1.7(0.2-13.5)$ \\
\hline$>9$ weeks & 8 & 8 & 1 & 1 \\
\hline
\end{tabular}

${ }^{*}$ For variables showing significant association during multivariate analysis at $p<0.05$.

\section{Discussion}

The present study determined the prevalence, key management outcomes, and factors associated with the management outcomes of EPs treated in Central Tigray region of Ethiopia. We found that the prevalence of EP was recorded in 5:1000 deliveries $(0.5 \%)$, a finding consistent with studies in Guinea (0.41\%) [9], and Papua New Guinea (0.6\%) [16]; although different globally, EP accounts for almost $2 \%$ [8, 9], South Africa (2.2\%) [12], and Nigeria (1.1\%) [13]. These variations could be linked to differences in environmental conditions (e.g., exposure to toxic materials), the prevalence of related infections (e.g., sexually transmitted infections), and social lifestyle (e.g., smoking). Evidence shows that smoking and infection could affect the transportation of an oocyte and embryo through the tube by decreasing the density of cilia.

The majority of women with EP (41.8\%) were in the 2630 years age group. This is comparable with the findings of studies conducted in India (71\%) and Nigeria (36\%) [3, 17]. This could partly be because of the maximum level of sexual activity and fertility of the women in this age period. This connotes the special need to have regular sexual and reproductive health check-ups in these age groups. The present study found that pelvic infection was observed in 24 women diagnosed with EP and had the following common symptoms: abdominal pain, vaginal bleeding, and Amenorrhea. This is similar to the study conducted in Ethiopia [7, 18, 19]. Prevention, or at least diagnosis and treatment, of pelvic infections would warrant primary care visits. Few other identified risk factors are modifiable.

More than one-third of the women admitted with EP (38\%) had an unfavorable outcome in the present study, and this was higher than a study conducted in Adigrat, Ethiopia [7]. This could partly be due to the differences in the ways that health facilities are set up in the two localities: Adigrat hospital was established many years before both hospitals in the current study setting and is also nearer to Ayder/ Mekele hospital, the most equipped hospital in the region.

In this study high rate of oophorectomy (21.1\%), this is similar to the study conducted in Ethiopia [20] and Boston Medical Center [21]. This high rate of oophorectomy was due to inability to achieve hemostasis without removal of ovary,

Urban residence and normal hemoglobin levels at presentation were the enabling factors linked with favorable outcomes of EP management in the present study. About $90 \%$ of hospitals in Ethiopia are located in urban areas despite more than $80 \%$ of the population living in rural areas, and it is unfortunately not surprising to see urban resident women having a more favorable outcome. Furthermore, the level of literacy among women living in urban 
areas is far better than their rural counterparts, and this may influence the early presentation to EP care and treatment, which is supported by research in Ethiopia and India [8, 18]. Consistent with other findings [1, 22], women diagnosed with EP and anemia were also at higher risk of unfavorable EP outcome management. Evidence suggest that endometriosis [23] and PID [24], leading to anemia, are known risk factors for adhesions and ectopic pregnancy. Additionally, the terminal ileum, sigmoid colon, and caecum were the parts of the gastrointestinal tract most commonly involved. The rectum was the source of bleeding in only one case.

The current study set out to comprehensively assess the magnitude of EP, its management outcomes, and related factors for EP management outcomes. However, there were some limitations that need highlighting. First, there was some data incompleteness linked to incomplete information, loss of patient cards, and illegible handwriting on patients' cards, gynecology registration books, and operation room records. Concerning this, we could not assess the details of sociodemographic variables and some important variables for some patients. Second, given it was a health facilitybased study, findings of the study may not be generalizable to the general population. Third, because the sample size was small $(n=79)$, some of the confidence intervals in the bivariate and multivariate logistic regression were rather wide. Fourth, the fact that women do not seek medical care in Ethiopia for many pregnancies in the first trimester limits the measurement, and that clinical or chemical pregnancy rate is not generally available, while surgeries for ectopic pregnancy and deliveries are much easier to identify.

\section{Conclusions}

The magnitude of EP in our study was similar to the global rate but more than one-third of women with ectopic pregnancies had an unfavorable maternal outcome. The delayed presentation with ruptures causes a major challenge to the reproductive performance of women and leads to maternal morbidity and mortality. This calls for an early checkup to improve the diagnosis, care, and treatment of EP and its management outcomes in developing countries like Ethiopia. Women living in rural areas and having anemia during pregnancy should seek special attention in the management of EP. We also recommend improving the data management of hospitals. The health care providers should document patients' information clearly on their cards. A large scale study with primary data of adequate sample size is required to assess the full picture of EP.

\section{Abbreviations}

$\begin{array}{ll}\text { ANC: } & \text { Antenatal care } \\ \text { BP: } & \text { Diastolic blood pressure } \\ \text { C/S: } & \text { Cesarean section } \\ \text { DES: } & \text { Diethylstilbestrol } \\ \text { E.C: } & \text { Ethiopian calendar } \\ \text { EDHS: } & \text { Ethiopian Demographic Health Survey } \\ \text { EP: } & \text { Ectopic pregnancy }\end{array}$

GP: $\quad$ General practitioner

GYN/OBS: Gynecology and obstetrics

HCG: Human chorionic gonadotrophic hormone

HIV: $\quad$ Human immunodeficiency virus

HP: $\quad$ Heterotopic pregnancy

IUD: Intrauterine device

OC: $\quad$ Oral contraceptives

${ }^{\circ} \mathrm{C}$ : $\quad$ Degree Celsius

Km: Kilometer

M: $\quad$ Meter

PID: $\quad$ Pelvic inflammatory disease

PNG: $\quad$ Papua New Guinea

RH: $\quad$ Reproductive health

RVI: $\quad$ Retroviral infection

SD: $\quad$ Standard deviation

STDs: $\quad$ Sexually transmitted diseases

SPSS: $\quad$ Statically Package for Social Science

TVUS: Transvaginal ultrasound

UK: $\quad$ United Kingdom

U/S: Ultrasound

WHO: World Health Organization.

\section{Data Availability}

The datasets analyzed in the study are available from the corresponding author upon request.

\section{Ethical Approval}

Ethical clearance was obtained from the Aksum University College of Health Science (CHS) ethical review committee, and permission letter was obtained from the Aksum University Comprehensive and Specialized Hospital (AKUCSH) and Saint Marries General Hospital before data collection. The patients' clinical records were reviewed anonymously before accessed all data, and confidentiality of the data was kept at each step of data collection and processing. Any identifier information was excluded during data collection.

\section{Conflicts of Interest}

The authors have declared that no competing interests exist.

\section{Authors' Contributions}

ETB, TGG, and KK designed the study. TGG performed statistical analysis. TGG and HAG $s$ drafted the paper. All authors contributed to writing the paper. All read and approved the final paper.

\section{Acknowledgments}

We are highly indebted to all participants of the study, supervisors of data collection, and data collectors for their worthy efforts and participation in this study. We are also thankful for administrative bodies at all levels who endorsed us to undertake this study. 


\section{Supplementary Materials}

Questionnaire 1. (Supplementary Materials)

\section{References}

[1] V. N. Sivalingam, W. C. Duncan, E. Kirk, L. A. Shephard, and A. W. Horne, "Diagnosis and management of ectopic pregnancy," The Journal of Family Planning and Reproductive Health Care, vol. 37, no. 4, pp. 231-240, 2011.

[2] G. Ganitha and G. Anuradha, "A study of incidence, risk factors, clinical profile and management of 50 cases of ectopic pregnancy in a tertiary care teaching hospital," International Journal of Reproduction, Contraception, Obstetrics and Gynecology, vol. 4, p. 1336, 2017.

[3] A. Panti, B. A. Tanko, A. Yakubu, S. C. Egondu, N. E. Ikechukwu, and O. O. lukman, "Ectopic pregnancy at Usmanu Danfodiyo University Teaching Hospital Sokoto: a ten year review," Annals of Nigerian Medicine, vol. 6, no. 2, p. 87, 2012.

[4] T. A. Christiane, D. Justin, T. Blaise, K. Victor, O. Serge, and T. Issifou, "Risk factors and prognosis of ruptured ectopic pregnancy in University Hopital of Benin," International Journal of Gynecology \& Clinical Practices, vol. 2, no. 1, 2015.

[5] J. Coste, J. Bouyer, S. Ughetto et al., "Ectopic pregnancy is again on the increase. Recent trends in the incidence of ectopic pregnancies in France (1992-2002)," Human Reproduction, vol. 19, no. 9, pp. 2014-2018, 2004.

[6] N. Goyaux, R. Leke, N. Keita, and P. Thonneau, "Ectopic pregnancy in African developing countries," Acta Obstetricia et Gynecologica Scandinavica, vol. 82, no. 4, pp. 305-312, 2003.

[7] D. Abebe, D. Tukue, A. Aregay, and L. Gebremariam, "Magnitude and Associated Factors with Ectopic Pregnancy Treated in Adigrat Hospital, Tigray Region, Northern Ethiopia," International Journal of Pharmaceutical Sciences and Research, vol. 7, no. 1, pp. 30-39, 2017.

[8] S. Marwah, K. U. Rani, and A. Mishra, "An audit of extrauterine pregnancy in a tertiary care facility in Northern India," International Journal of Reproduction, Contraception, Obstetrics and Gynecology, vol. 6, no. 11, p. 5025, 2017.

[9] P. Thonneau, Y. Hijazi, N. Goyaux, T. Calvez, and N. Keita, "Ectopic pregnancy in Conakry, Guinea," Bulletin of the World Health Organization, vol. 80, no. 5, pp. 365-370, 2002.

[10] S. Shaikh, S. Jampala, S. S. Devi, and M. Mallika, "A study of ectopic pregnancy in a tertiary care teaching hospital," Indian Journal of Obstetrics and Gynecology Research, vol. 3, no. 2, pp. 132-136, 2016.

[11] A. Igwegbe, G. Eleje, and B. Okpala, "An appraisal of the management of ectopic pregnancy in a Nigerian tertiary hospital," Annals of Medical and Health Sciences Research, vol. 3, no. 2, pp. 166-170, 2013.

[12] D. K. Nzaumvila, I. Govender, and G. A. Ogunbanjo, "An audit of the management of ectopic pregnancies in a district hospital, Gauteng, South Africa," African Journal of Primary Health Care \& Family Medicine, vol. 10, no. 1, pp. 1-8, 2018.

[13] M. S. Yeasmin, M. J. Uddin, and E. Hasan, "A clinical study of ectopic pregnancies in a tertiary care hospital of Chittagong, Bangladesh," Chattagram Maa-O-Shishu Hospital Medical College Journal, vol. 13, no. 3, pp. 1-4, 2015.

[14] Centre, IDR and I Network, Population and health in developing countries: population, health and survival at INDEPTH sites, vol. 1, IDRC, 2002.
[15] E. J. Keller, Revolutionary Ethiopia: from empire to people's republic, vol. 646, Indiana University Press, 1991.

[16] N. N. Hamura, J. W. Bolnga, R. Wangnapi, A. W. Horne, S. J. Rogerson, and H. W. Unger, "The impact of tubal ectopic pregnancy in Papua New Guinea-a retrospective case review," BMC Pregnancy and Childbirth, vol. 13, no. 1, pp. 1-8, 2013.

[17] D. Panchal, G. Vaishnav, and K. Solanki, "Study of Management in Patient with Ectopic Pregnancy," Infection, vol. 33, p. 55, 2011.

[18] U. Gerema, T. Alemayehu, G. Chane, D. Desta, and A. Diriba, "Determinants of ectopic pregnancy among pregnant women attending referral hospitals in southwestern part of Oromia regional state, Southwest Ethiopia: a multi-center case control study," BMC Pregnancy and Childbirth, vol. 21, no. 1, pp. 1-8, 2021.

[19] A. N. Negewo, "Prevalence and management outcome of ectopic pregnancy in Adama Hospital Medical College, East Shoa Zone, Oromia Region, Ethiopia," EC Gynaecology, vol. 8, pp. 844-850, 2019.

[20] G. Diriba, G. Tol, and D. Hiko, "Prevalence and management outcome of ectopic pregnancy: a three year retrospective study in Adama hospital medical college east shoa zone, Oromia region, Ethiopia," BMC Pregnancy and Childbirth, vol. 21, no. 1, pp. 1-8, 2013.

[21] L. D. Michelis, J. A. Politch, and W. Kuohung, "Factors associated with oophorectomy in patients with suspected ovarian torsion," Journal of Gynecologic Surgery, vol. 37, no. 3, pp. 236-240, 2021.

[22] H. Akkaya and G. Uysal, "Can hematologic parameters predict treatment of ectopic pregnancy?," Pakistan Journal of Medical Sciences, vol. 33, no. 4, pp. 937-942, 2017.

[23] P. Parasar, P. Ozcan, and K. L. Terry, "Endometriosis: epidemiology, diagnosis and clinical management," Current Obstetrics and Gynecology Reports, vol. 6, no. 1, pp. 34-41, 2017.

[24] C.-C. Huang, C. C. Huang, S. Y. Lin et al., "Association of pelvic inflammatory disease (PID) with ectopic pregnancy and preterm labor in Taiwan: a nationwide population-based retrospective cohort study," PLoS One, vol. 14, no. 8, article e0219351, 2019. 